\title{
Percutaneous Tricuspid Valve Replacement Two Cases of Valve-in-Valve and Valve-in-Ring
}

\author{
Steve Zhou, BS ${ }^{1 *}$, Jamil Aboulhosn, MD² \\ 1 David Geffen School of Medicine, University of California Los Angeles, Los Angeles, California, USA \\ ${ }^{2}$ Ahmanson/University of California Los Angeles Adult Congenital Heart Disease Center, Los Angeles, California, USA
}

\begin{abstract}
Tricuspid valve (TV) replacement via transcatheter techniques is feasible but not yet widely utilized. The two cases reported here describe transcatheter replacement of a failing bioprosthetic TV and a failing native valve that previously underwent surgical annuloplasty repair. The first case was a 26-year-old male with Ebstein's anomaly who underwent surgical TV replacement with a 33-mm Hancock bioprosthesis 15 years prior to transcatheter intervention. The valve had become stenotic and regurgitant and was successfully replaced with a Melody valve mounted on a 22-mm Ensemble balloon-in-balloon system. The second case was a 59-year-old female with rheumatic heart disease who had undergone prior surgical TV repair with a $30-\mathrm{mm}$ Edwards incomplete annuloplasty ring. Her repaired valve eventually became severely regurgitant, and she underwent successful transcatheter valve replacement using a 29-mm Sapien XT valve. She exhibited paravalvular regurgitation necessitating vascular plug implantation. These two cases highlight the potential utility and limitations of commercially available transcatheter valves in the tricuspid position. Moreover, this report compares and contrasts the procedural nuances of TV replacement within a complete bioprosthetic valve ring versus an open annuloplasty ring.

Copyright ( 2017 Science International Corp.
\end{abstract}

\section{Key Words:}

Tricuspid valve stenosis • Tricuspid valve insufficiency • Ebstein's anomaly - Transcatheter valve replacement •

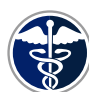

Fax +1 2037853346

E-Mail: jshd@scienceinternational.org

http://structuralheartdisease.org/ (c) 2017 Journal of Structural Heart Disease Published by Science International Corp. ISSN 2326-4004

Accessible online at: http://structuralheartdisease.org/
Right-sided heart failure • Cardiac valve annuloplasty • Heart valve prosthesis

\section{Introduction}

Native tricuspid valve (TV) dysfunction is associated with both congenital and acquired heart conditions. Conditions such as severe pulmonary hypertension or right ventricular (RV) outflow obstruction can lead to elevated RV systolic pressure, commonly causing RV and TV annular dilation and subsequent tricuspid regurgitation (TR). Patients with congenital RV outflow dysfunction, such as repaired Tetralogy of Fallot with pulmonic stenosis and/or regurgitation, commonly develop secondary TR. Other congenital conditions associated with primary TV dysfunction include Ebstein's anomaly, with regurgitation occurring due to dysplastic tricuspid leaflets. Although TR may be well tolerated for decades, eventual clinical sequelae usually emerge. These most commonly involve progressive RV dysfunction, the development of ventricular and supraventricular arrhythmias, and elevated central venous pressure leading to multi-organ congestion.

TV repair techniques include the placement of annular rings as well as more complex operations to relocate or augment TV leaflets in patients with Ebstein's anomaly. Repair is usually successful in the short and intermediate term, but up to $25 \%$ of

* Corresponding Author:

Steve Zhou, BS

David Geffen School of Medicine

University of California Los Angeles

885 Tiverton Drive, Los Angeles, California 90095, USA

Tel: +1 650796 1026; Fax: +1 310825 9012; E-Mail: szhou@mednet.ucla.edu 
patients develop moderate or severe regurgitation within 5 years $[1,2]$. In patients with congenital forms of TR, surgical replacement may be necessary at a young age; longer-lasting mechanical valves would require placing young patients on anticoagulants, and yet bioprosthetic valves commonly require replacement within 10-15 years [3,4]. Use of biological valves early in life most certainly predicts multiple reoperations as they begin to fail, and repeat surgery is associated with increased morbidity and mortality [5].

Percutaneous TV replacement may be a viable alternative to surgical reoperation. This procedure has been well documented in the aortic and pulmonary valve positions, and there is a growing body of evidence for the use of both the Melody (Medtronic Inc.) and Sapien (Edwards Lifesciences) valves in failing TV rings and bioprostheses [6]. Although the procedural success rate is high with immediate hemodynamic benefits, there is paucity of long-term data. In terms of recurrent TR post annuloplasty, successful utilization of transcatheter valves is limited to isolated case reports and small case series $[7,8,9]$. The present report details two cases of transcatheter TV replacement-one within a failing bioprosthesis and the other in a regurgitant native valve with an incomplete annuloplasty ring-and seeks to highlight the differences in technique, device selection, and procedural outcomes.
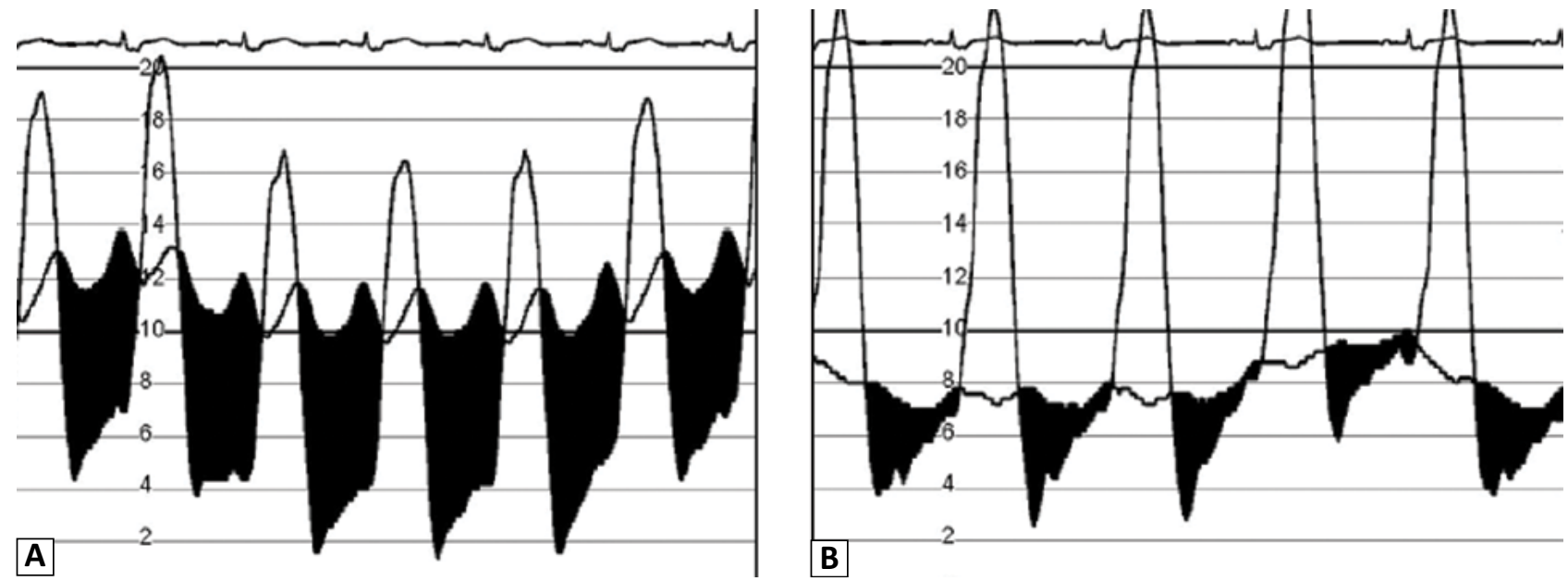

Figure 1. Panel A. Pre-deployment mean RV-pulmonary artery gradient measurement of $6 \mathrm{mmHg}$, Fick cardiac output of $4.45 \mathrm{~L} / \mathrm{min}$, and estimated TV area by the Gorlin formula of $0.97 \mathrm{~cm}^{2}$. Panel B. Post-deployment mean RV-pulmonary artery gradient measurement of $2 \mathrm{mmHg}$.

Journal of Structural Heart Disease, April 2017

Volume 3, Issue 2:55-61 
Following diagnostic catheterization, an Amplatzer super-stiff (St. Jude Inc.) was placed in the right pulmonary artery, and a PTS-X 30-mm balloon (Numed Inc.) was inflated across the tricuspid bioprosthesis with a 21-mm waist noted (Figure 2A). A Melody valve was then mounted on a $22-\mathrm{mm}$ Ensemble balloon-in-balloon delivery system and deployed within the Hancock II bioprosthesis (Figure 2B). Subsequent hemodynamic assessment showed a reduction in the mean inflow diastolic gradient to $2 \mathrm{mmHg}$ (Figure 1B) without evidence of intra- or paravalvular leak (PVL). The patient was extubated in the catheterization laboratory and discharged to home the following day on daily $325 \mathrm{mg}$ aspirin therapy. A post-operative echocardiogram revealed a mean inflow gradient of $3.5 \mathrm{mmHg}$ and trace TR. The patient reported increased physical stamina and denied dyspnea on moderate exertion.

However, 8 months after the valve implantation, a routine echocardiogram demonstrated an increase in the diastolic transvalvular mean Doppler gradient to $11 \mathrm{mmHg}$, suggestive of valve stenosis. The valve was not well visualized, but there was clinical suspicion of a possible thrombotic etiology. The patient was initiated on anticoagulation with rivaroxaban 20 $\mathrm{mg}$ daily with a reduction in the mean diastolic gra- dient by Doppler to $4 \mathrm{mmHg}$ within 3 weeks, and the valve has continued to function well 14 months postimplantation.

\section{Case 2: Failing Native Valve with Incomplete An- nuloplasty Ring}

A 59-year-old female with a history of atrial fibrillation and rheumatic mitral valve (MV) stenosis underwent bioprosthetic MV replacement and the Maze procedure at 46 years of age. At 57 years of age, she received a redo MV replacement for prosthetic valve stenosis and TV repair with a $30-\mathrm{mm}$ Edwards incomplete annuloplasty ring for severe TR. She developed high-grade atrioventricular block and also received a transvenous permanent dual chamber pacemaker with an atrial lead and a ventricular lead placed in a lateral cardiac vein via the coronary sinus to avoid crossing the TV. Within 1 year, she developed heart failure symptoms and required up-titration of diuretic therapy and home intravenous dobutamine. She had progressive renal failure and severe recurrent TR requiring hospitalization and initiation of multiple inotropic medications and continuous veno-venous dialysis. She was also diagnosed with congestive hepatic cirrhosis. She was evaluated for surgical valve re-
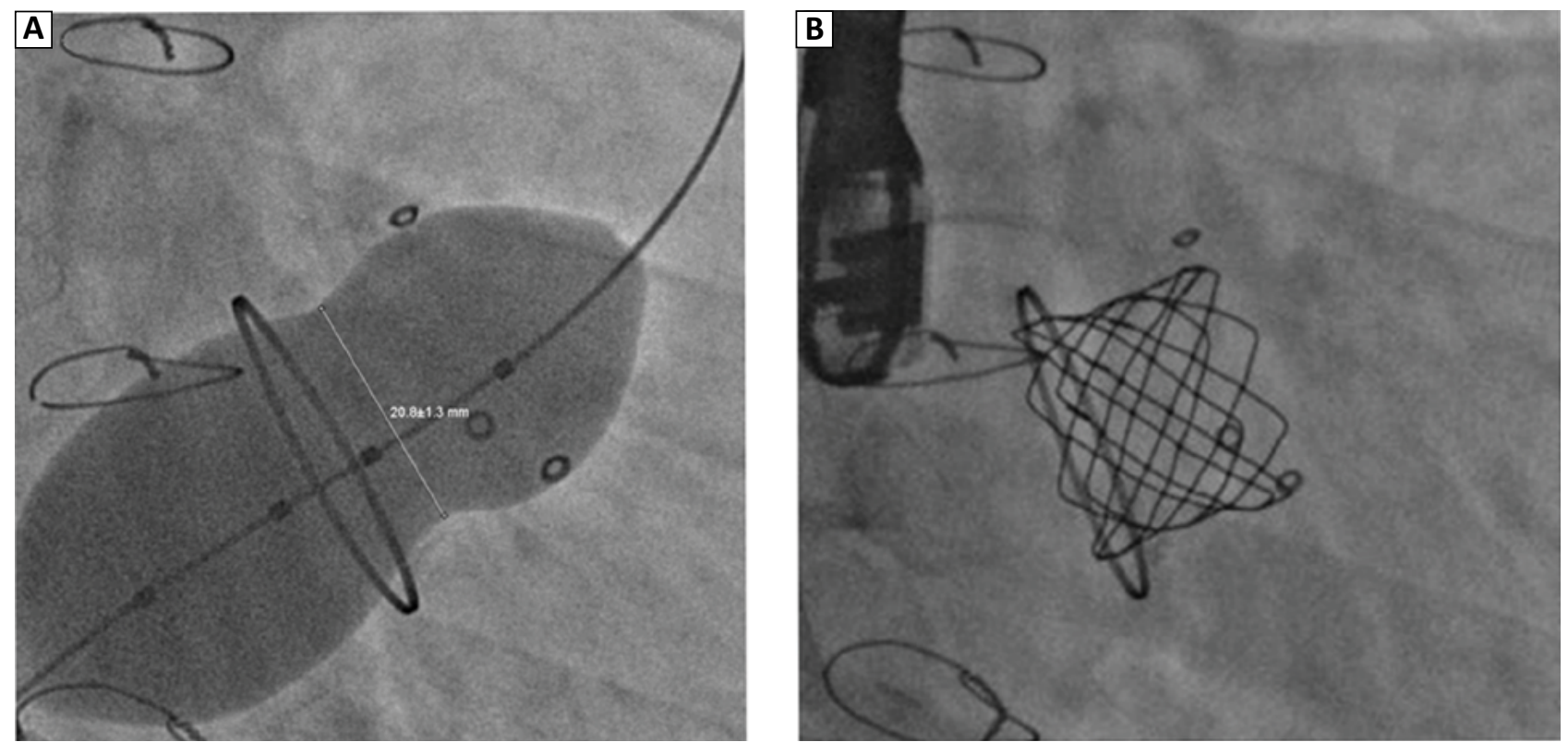

Figure 2. Panel A. Balloon inflation across the stenotic prosthesis indicated a 21-mm waist. Panel B. Post-placement of a 22-mm Melody valve. 
placement and multi-organ transplantation but was felt to not be a candidate for either. Percutaneous TV replacement was considered as a potential palliation.

The patient underwent transcatheter TV implantation using a 29-mm Edwards Sapien XT valve without pre-stenting of the ring. The procedure was guided by TEE and fluoroscopy and performed under general anesthesia (Figure 3A). Via a femoral venous approach, a 30-mm $\times$ 4-cm PTS-X balloon (Numed) was inflated across the annuloplasty ring to reveal a 27-28 mm waist (Figure 4A) with evidence of mild to moderate residual regurgitation within the medial "open" portion of the ring (Figure 3B). Right coronary angiography showed no evidence of compression. A 29-mm Edwards Sapien XT valve and delivery system were introduced into the tricuspid position. The valve was deployed with rapid pacing at $160 \mathrm{bpm}$ using the existing permanent pacemaker (Figure 4B). Following valve deployment, TEE imaging demonstrated moderate medial PVL and mild lateral PVL (Figure $3 C$ ). The medial PVL was successfully crossed with a guide wire and catheter, and two Amplatzer vascular plugs (AVP II, St. Jude Medical) were successfully placed, reducing the PVL to mild (Figure 3D).

The patient tolerated the procedure well and was weaned from all pressor support within 1 week, remaining hemodynamically stable thereafter. Her renal function improved dramatically, and she was able to discontinue dialysis 1 month post-intervention. Follow-up transthoracic echocardiograms showed mild TR with no stenosis 8 months post-intervention. Her functional capacity improved from New York Heart
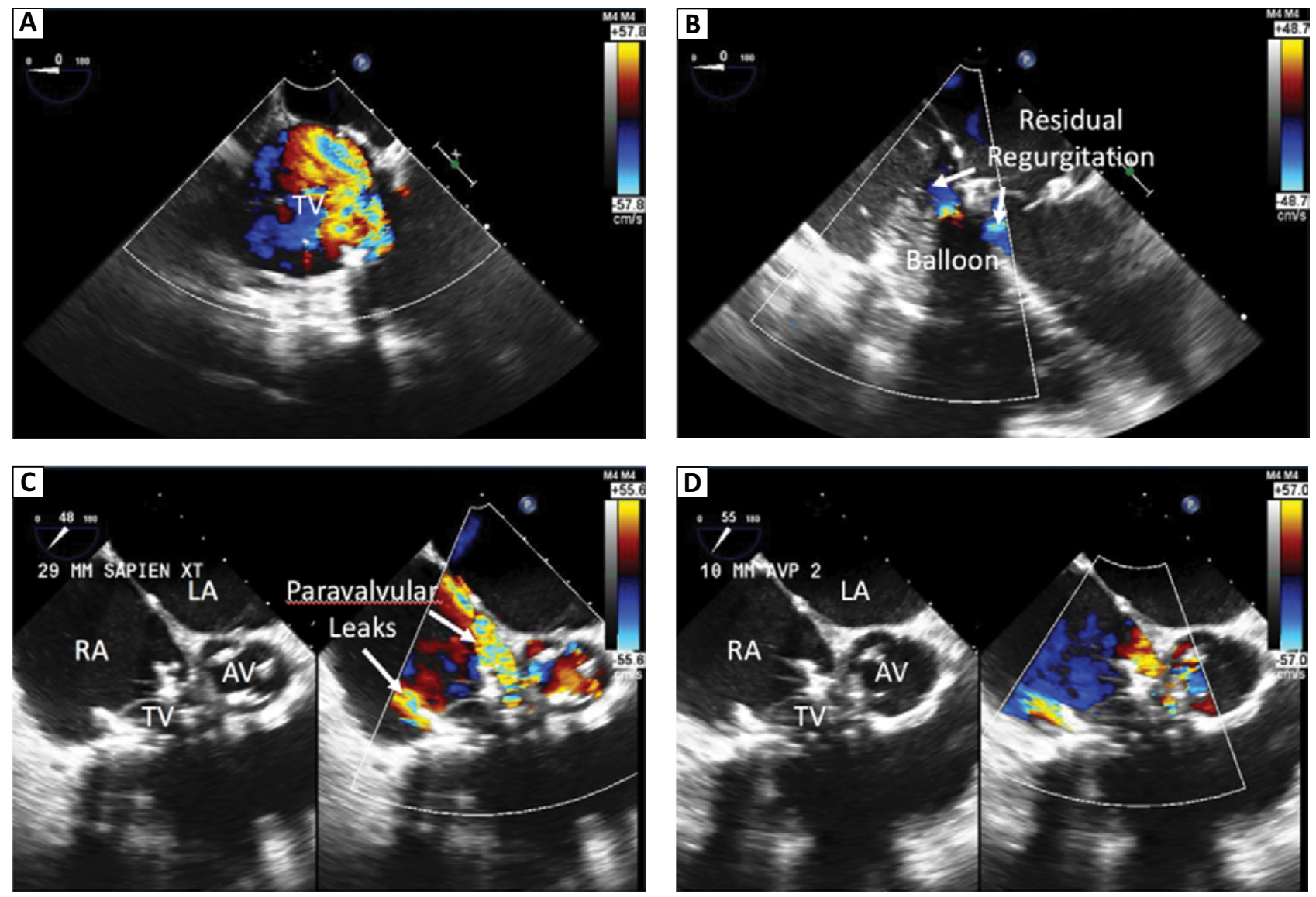

Figure 3. Transesophageal Doppler echocardiographic images. Panel A. Tricuspid valve color Doppler pre-deployment demonstrating severe regurgitation. Panel B. Balloon inflation across the native valve demonstrated residual medial regurgitation in the open part of the annuloplasty ring. Panel C. Post-deployment of a 29-mm Sapien XT valve showed moderate medial and mild lateral paravalvular leak (PVL). PanelD. Post-deployment of Amplatzer vascular plugs medially resulted in a reduction of PVL. No central regurgitation was noted. $\mathrm{RA}=$ right atrium; $\mathrm{LA}=$ left atrium; TV = tricuspid valve; $\mathrm{AV}=$ aortic valve. 
Association (NYHA) class IV prior to the procedure to NYHA class II within 3 months. However, increasing severity of the lateral PVL precipitated worsening heart failure symptoms and volume overload. Therefore, she underwent repeat catheterization 10 months after valve replacement with successful occlusion of the lateral PVL under intracardiac echo guidance (Figure 5A). Follow-up echocardiography at 3 and 6 months showed stable valve function and mild lateral and medial PVL (Figure 5B).
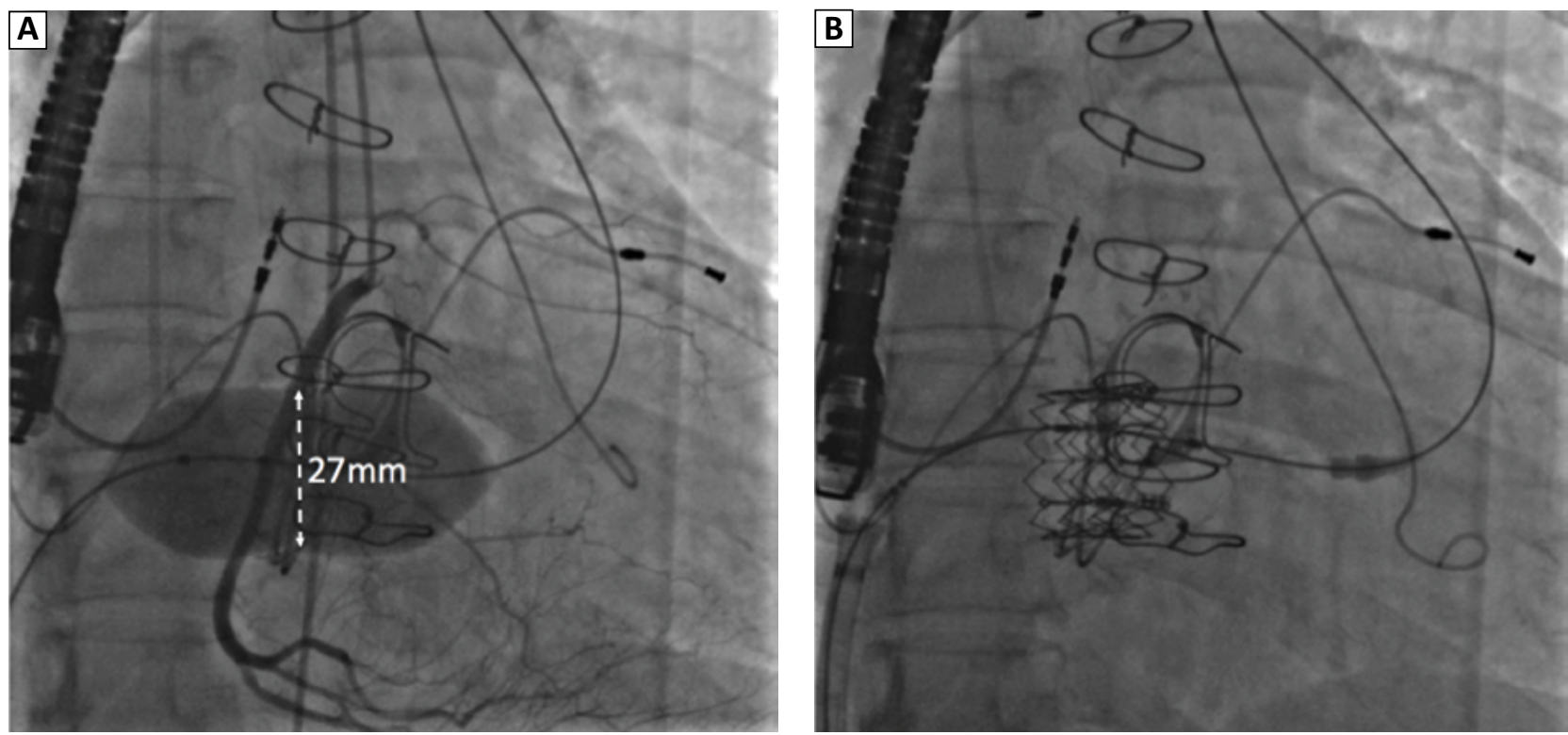

Figure 4. Panel A. Balloon inflation across the annuloplasty ring indicated a 27-mm waist. Panel B. Post-placement of a 29-mm Sapien XT valve.
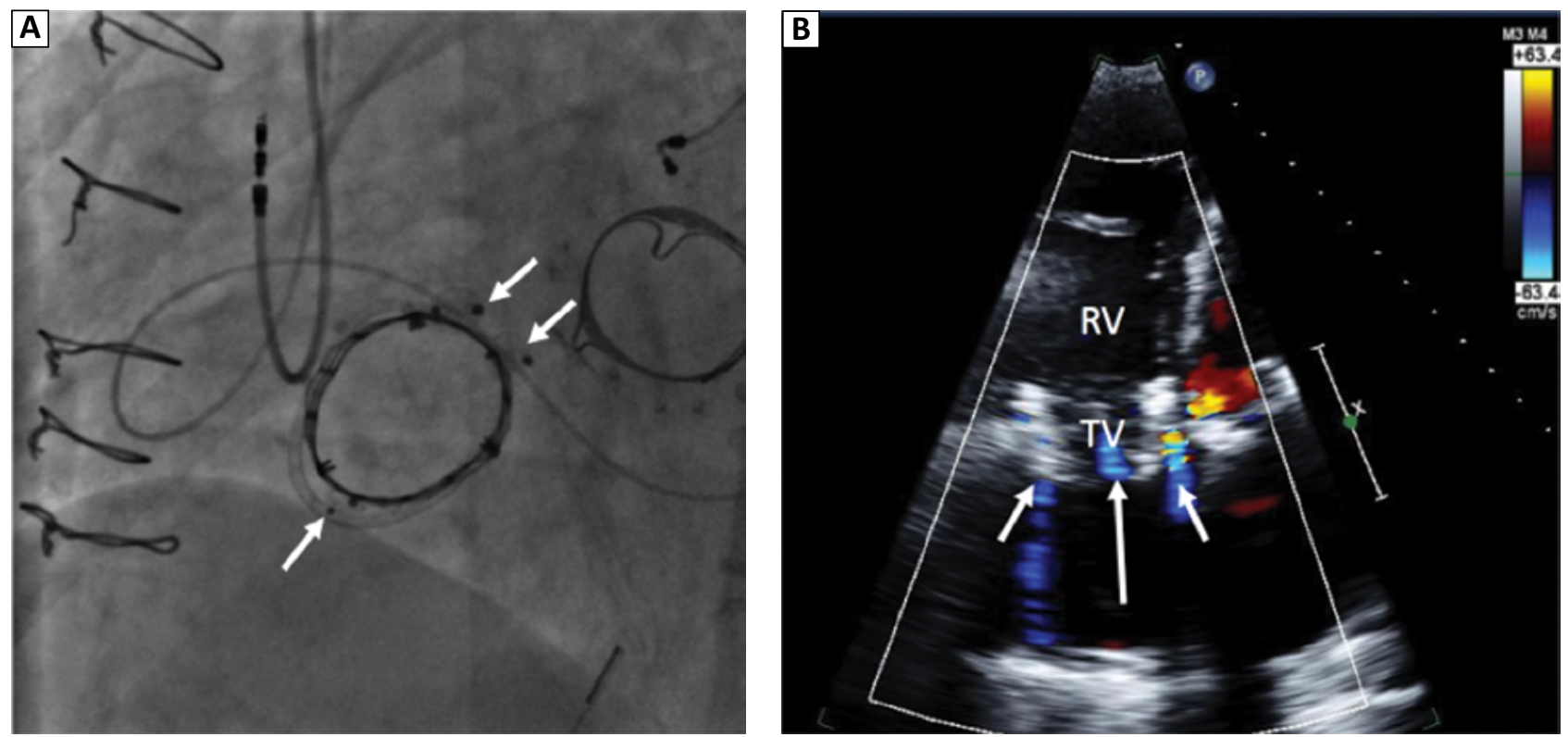

Figure 5. Panel A. Post-deployment of additional Amplatzer vascular plugs to treat worsening lateral paravalvular leak (PVL) in addition to medial plugs from initial catheterization. Panel B. Transthoracic echocardiogram with Doppler post-deployment of additional vascular plugs showed reduced PVL. Trace central regurgitation was noted. RV = right ventricular; TV = tricuspid valve. 


\section{Discussion}

Although there is an increasing number of reports of successful transcatheter deployment of biological valves in the tricuspid position, this is not yet standard of care. In 2011, a study of 15 patients from eight medical centers reported successful deployment in 12 patients without complication [10]. A more recent study of 10 patients, including five with Ebstein's anomaly, reported only one complication of valve migration post-deployment [11]. The largest experience to date is a multi-center database of TVIV implants detailing the outcomes and 1-year follow-up of 156 patients with TVIV, which demonstrated no procedural mortality and excellent short- to medium-term outcomes [6]. Our two cases involved specific complexities of the procedure and post-implantation outcome, demonstrating our approach to their management.

Among these patients, it is important to identify the primary diagnosis because Ebstein's anomaly is associated with complex hemodynamics. Patients with Ebstein's anomaly often suffer from RV dysfunction and restrictive diastolic function and may have undergone numerous surgical repairs and replacements. Moreover, they are at increased risk for both atrial and ventricular arrhythmias [12]. As the morbidity and mortality risk associated with surgical TV replacement is often high [5], the excellent short- and intermediate-term outcomes of trans-catheter valve replacement make it a viable alternative.

Surgical TV replacement is most often performed using bioprosthetic valves. There is limited experience with mechanical valves in this position, and there is a concern for a higher risk of valve thrombosis in the tricuspid position [13]. It has traditionally been recognized that the primary mode of bioprosthetic TV failure is structural deterioration with resultant calcification and progressive stenosis and regurgitation. However, recent studies show that bioprosthetic valve thrombosis (BPVT) is common and a more underdiagnosed mode of failure than originally thought $[14,15]$. The estimated prevalence of BPVT in the tricuspid position is $12 \%$, resulting in early failure of the valve usually occurring within 5 years of implantation. BPVT of a tricuspid bioprosthesis is usually insidious in presentation and re- sults from an elevation in central venous pressure, which symptomatically is much less dramatic than left-sided valve thrombosis with a resultant elevation in left atrial pressure and pulmonary edema [13, 16]. Pathognomonic signs of BPVT include an acute rise in transvalvular gradient of more than $50 \%$ over baseline in the short- to medium-term, paroxysmal atrial fibrillation, increased cusp thickness, decreased cusp mobility, and sub-therapeutic international normalized ratio [16]. BPVT was highly likely in Case 1, in which there was a sharp increase in valve gradient $(>50 \%)$ within 8 months after Melody valve placement. The initiation of anticoagulation resulted in a dramatic improvement in valve gradient, which provides additional support for the assertion that this was early BPVT. The use of non-vitamin K antagonists for anticoagulation following bioprosthetic valve replacement has not been well studied but in this case proved effective.

Percutaneous valve replacement of native TVs presents with additional challenges due to the lack of secure circumferential scaffolding provided by surgical bioprostheses. The native annulus is highly distensible and is not a stable landing zone for balloon-dilated transcatheter valves. In patients with annuloplasty bands or rings of the TV, a transcatheter valve-in-ring approach is feasible. Case 2 demonstrates that such an approach is possible in patients with incomplete annuloplasty rings from previous TV repair.

It should be noted that an incomplete ring annuloplasty does not supply the same level of support as a previously placed bioprosthetic valve. PVL is common and should be expected, especially in the medial open part of the ring. However, this can be readily treated with transcatheter vascular plug/ occluder deployment at the time of valve implantation. PVL can be progressive, as in Case 2, and may require additional vascular plug placement during follow-up.

Additionally, it is important to recognize that the reduced radial support along the open portion of the existing ring could ostensibly lead to deformation of the bioprosthetic valve. Sufficient loss of circular shape could prevent the leaflets from coapting as originally designed, potentially resulting in regurgitation. However, despite the loss of the circular shape in Case 2, there was minimal intravalvular regurgitation. 
Paravalvular regurgitation is expected and was adequately treated with vascular plugs.

\section{Conclusion}

In addition to exhibiting immediate post-interventional improvements and relief of symptoms without complication, both patients have remained stable in the intermediate term. One patient likely developed Melody valve thrombosis, which was effectively treated with oral anticoagulation. Pending additional long-term follow-up, these cases provide evidence of substantial and versatile benefits of a transcatheter approach to TV replacement, both in bioprosthetic and native TV post-surgical annuloplasty.

\section{Conflict of Interest}

The authors have no conflict of interest relevant to this publication.

\section{Comment on this Article or Ask a Question}

\section{References}

1. McCarthy PM, Bhudia SK, Rajeswaran J, Hoercher KJ, Lytle BW, Cosgrove DM, et al. Tricuspid valve repair: Durability and risk factors for failure. J Thorac Cardiovasc Surg. 2004;127:674-685. DOI: 10.1016/j. jtcvs.2003.11.019

2. Jeganathan R, Armstrong S, Al-Alao B, David T. The risk and outcomes of reoperative tricuspid valve surgery. Ann Thorac Surg. 2013;95:119-124. DOI: 10.1016/j. athoracsur.2012.08.058

3. Marquis-Gravel G, Bouchard D, Perrault LP, Pagé $P$, Jeanmart $H$, Demers $P$, et al. Retrospective cohort analysis of 926 tricuspid valve surgeries: Clinical and hemodynamic outcomes with propensity score analysis. Am Heart J. 2012;163:851-858. DOI: 10.1016/j.ahj.2012.02.010

4. Brown ML, Dearani JA, Danielson GK, Cetta F, Connolly HM, Warnes CA, et al. Comparison of the outcome of porcine bioprosthetic versus mechanical prosthetic replacement of the tricuspid valve in the Ebstein anomaly. Am J Cardiol. 2009;103:555-561. DOI: 10.1016/j.amjcard.2008.09.106

5. Iscan ZH, Vural KM, Bahar I, Mavioglu L, Saritas A. What to expect after tricuspid valve replacement? Long-term results. Eur J Cardiothorac Surg. 2007;32:296-300. DOI: 10.1016/j.ejcts.2007.05.003

6. McElhinney DB, Cabalka AK, Aboulhosn JA, Eicken A, Boudjemline $Y$, Schubert $S$, et al. Transcatheter tricuspid valve-invalve implantation for the treatment of dysfunctional surgical bioprosthetic valves:
An international multicenter registry study. Circulation. 2016;133:1537-1539. DOI: 10.1161/CIRCULATIONAHA.115.019353

7. Bouleti $C$, Himbert $D$, Brochet $E$, Ou $P$, lung $B$, Nejjari $M$, et al. Transfemoral tricuspid valve-in-ring implantation using the edwards sapien xt valve: Oneyear follow-up. Circ Cardiovasc Interv. 2015;8:e02225. DOI: 10.1161/CIRCINTERVENTIONS.114.002225

8. Condado J, Leonardi R, Babaliaros V. Percutaneous tricuspid valve-in-ring replacement for the treatment of recurrent severe tricuspid regurgitation. Catheter Cardiovasc Interv. 2015;86:1294-1298. DOI: $10.1002 / \mathrm{ccd} .25980$

9. Kefer J, Sluysmans T, Vanoverschelde JL. Transcatheter sapien valve implantation in a native tricuspid valve after failed surgical repair. Catheter Cardiovasc Interv. 2014;83:841-845. DOI: 10.1002/ccd.25330

10. 10. Roberts $P A$, Boudjemline $Y$, Cheatham JP, Eicken A, Ewert P, McElhinney DB, et al. Percutaneous tricuspid valve replacement in congenital and acquired heart disease. J Am Coll Cardiol. 2011;58:117-122. DOI: 10.1016/j.jacc.2011.01.044

11. Godart F, Baruteau $A E$, Petit J, Riou JY, Sassolas F, Lusson JR. Transcatheter tricuspid valve implantation: A multicenter French study. Arch Cardiovasc Dis. 2014;107:583591. DOI: 10.1016/j.acvd.2014.07.051

12. Dearani JA, Mora BN, Nelson TJ, Haile DT, O'Leary PW. Ebstein anomaly review: What's now, what's next? Expert Rev Cardiovasc Ther. 2015;13:1101-1109. DOI:

\subsection{6/14779072.2015.1087849}

13. Throburn CW, Morgan JJ, Shanahan MX, Chang VP. Long-term results of tricuspid valve replacement and the problem of prosthetic valve thrombosis. Am J Cardiol. 1983;51:1128-1132. PMID: 6837458

14. Egbe AC, Pislaru SV, Pellikka PA, Poterucha JT, Schaff HV, Maleszewski JJ, et al. Bioprosthetic valve thrombosis versus structural failure: Clinical and echocardiographic predictors. J Am Coll Cardiol. 2015;66:2285-2294. DOI: 10.1016/j. jacc.2015.09.022

15. Reddy YN, Connolly HM, Ammash NM. Thrombotic obstruction of a Melody valve-in-valve used for prosthetic tricuspid stenosis. World J Pediatr Congenit Heart Surg. 2015;6:667-669. DOI: $10.1177 / 2150135115586270$

16. Pislaur SV, Pellikka PA, Schaff HV, Connolly HM. Bioprosthetic valve thrombosis: The eyes will not see what the mind does not know. J Thorac Cardiovasc Surg. 2015;149:e83-e86. DOI: 10.1016/j. jtcvs.2015.03.012

Cite this article as: Zhou S, Aboulhosn J. Percutaneous Tricuspid Valve Replacement. Structural Heart Disease. 2017;3(2):55-61. DOI: http://dx.doi. org/10.12945/j.jshd.2017.012.16 